\title{
Collective Tree Spanners in Graphs with Bounded Parameters
}

\author{
Feodor F. Dragan • Chenyu Yan
}

Received: 3 February 2006 / Accepted: 16 April 2008

(C) Springer Science+Business Media, LLC 2008

\begin{abstract}
In this paper we study collective additive tree spanners for special families of graphs including planar graphs, graphs with bounded genus, graphs with bounded tree-width, graphs with bounded clique-width, and graphs with bounded chordality. We say that a graph $G=(V, E)$ admits a system of $\mu$ collective additive tree $r$-spanners if there is a system $\mathcal{T}(G)$ of at most $\mu$ spanning trees of $G$ such that for any two vertices $x, y$ of $G$ a spanning tree $T \in \mathcal{T}(G)$ exists such that $d_{T}(x, y) \leq d_{G}(x, y)+r$. We describe a general method for constructing a "small" system of collective additive tree $r$-spanners with small values of $r$ for "well" decomposable graphs, and as a byproduct show (among other results) that any weighted planar graph admits a system of $O(\sqrt{n})$ collective additive tree 0 -spanners, any weighted graph with tree-width at most $k-1$ admits a system of $k \log _{2} n$ collective additive tree 0 -spanners, any weighted graph with clique-width at most $k$ admits a system of $k \log _{3 / 2} n$ collective additive tree (2w)-spanners, and any weighted graph with size of largest induced cycle at most $c$ admits a system of $\log _{2} n$ collective additive tree $(2\lfloor c / 2\rfloor \mathrm{w})$-spanners and a system of $4 \log _{2} n$ collective additive tree $(2(\lfloor c / 3\rfloor+1) \mathrm{w})$ spanners (here, $w$ is the maximum edge weight in $G$ ). The latter result is refined for weighted weakly chordal graphs: any such graph admits a system of $4 \log _{2} n$ collective additive tree $(2 w)$-spanners. Furthermore, based on this collection of trees, we derive a compact and efficient routing scheme for those families of graphs.
\end{abstract}

Results of this paper were partially presented at the ISAAC'05 conference [14].

F.F. Dragan $(\bowtie) \cdot$ C. Yan

Algorithmic Research Laboratory, Department of Computer Science, Kent State University, Kent,

$\mathrm{OH}$ 44242, USA

e-mail: dragan@cs.kent.edu

C. Yan

e-mail: cyan@cs.kent.edu 
Keywords Spanners · Tree spanners $\cdot$ Graph distance $\cdot$ Balanced separator $\cdot$ Graph decomposition · Tree-width · Clique-width · Planar graphs $\cdot c$-Chordal graphs ·

Message routing $\cdot$ Efficient algorithms

\section{Introduction}

Many combinatorial and algorithmic problems are concerned with the distance $d_{G}$ on the vertices of a possibly weighted graph $G=(V, E)$. Approximating $d_{G}$ by a simpler distance (in particular, by tree-distance $d_{T}$ ) is useful in many areas such as communication networks, data analysis, motion planning, image processing, network design, and phylogenetic analysis. An arbitrary metric space (in particular a finite metric defined by a general graph) might not have enough structure to exploit algorithmically. Given a graph $G=(V, E)$, a spanning subgraph $H$ is called a spanner if $H$ provides a "good" approximation of the distances in $G$. More formally, for $t \geq 1$, $H$ is called a multiplicative $t$-spanner of $G[31,32]$ if $d_{H}(u, v) \leq t \cdot d_{G}(u, v)$ for all $u, v \in V$. If $r \geq 0$ and $d_{H}(u, v) \leq d_{G}(u, v)+r$ for all $u, v \in V$, then $H$ is called an additive $r$-spanner of $G$ [30]. The parameters $t$ and $r$ are called, respectively, the multiplicative and the additive stretch factors. When $H$ is a tree one has a multiplicative tree t-spanner [8] and an additive tree $r$-spanner [33] of $G$, respectively. For some recent results on sparse spanners and tree spanners of graphs we refer the reader to [17-19].

In this paper, we continue the approach taken in $[10,15,16,26]$ of studying collective tree spanners. We say that a graph $G=(V, E)$ admits a system of $\mu$ collective additive tree $r$-spanners if there is a system $\mathcal{T}(G)$ of at most $\mu$ spanning trees of $G$ such that for any two vertices $x, y$ of $G$ a spanning tree $T \in \mathcal{T}(G)$ exists such that $d_{T}(x, y) \leq d_{G}(x, y)+r$ (a multiplicative variant of this notion can be defined analogously). Clearly, if $G$ admits a system of $\mu$ collective additive tree $r$-spanners, then $G$ admits an additive $r$-spanner with at most $\mu(n-1)$ edges (take the union of all those trees), and if $\mu=1$ then $G$ admits an additive tree $r$-spanner. Note also that any graph on $n$ vertices admits a system of at most $n-1$ collective additive tree 0 -spanners (take $n-1$ Shortest-Path-trees rooted at different vertices of $G$ ). In particular, we examine the problem of finding "small" systems of collective additive tree $r$-spanners for small values of $r$ on special classes of graphs such as planar graphs, graphs with bounded genus, graphs with bounded tree-width, graphs with bounded clique-width, and graphs with bounded chordality.

Previously, collective tree spanners of particular classes of graphs were considered in $[10,15,16,26]$. Paper [16] showed that any unweighted chordal graph, chordal bipartite graph or cocomparability graph admits a system of at most $\log _{2} n$ collective additive tree 2 -spanners. These results were complemented by lower bounds, which say that any system of collective additive tree 1-spanners must have $\Omega(\sqrt{n})$ spanning trees for some chordal graphs and $\Omega(n)$ spanning trees for some chordal bipartite graphs and some cocomparability graphs. Furthermore, it was shown that any unweighted $c$-chordal graph admits a system of at most $\log _{2} n$ collective additive tree $(2\lfloor c / 2\rfloor)$-spanners and any unweighted circular-arc graph admits a system of two collective additive tree 2-spanners. Paper [15] showed that any unweighted 
AT-free graph (graph without asteroidal triples) admits a system of two collective additive tree 2-spanners, any unweighted graph having a dominating shortest path admits a system of two collective additive tree 3-spanners and a system of five collective additive tree 2-spanners, and any unweighted graph with asteroidal number an $(G)$ admits a system of $\operatorname{an}(G)(\operatorname{an}(G)-1) / 2$ collective additive tree 4-spanners and a system of an $(G)(\operatorname{an}(G)-1)$ collective additive tree 3-spanners. In paper [10], it was shown that no system of constant number of collective additive tree 1-spanners can exist for unit interval graphs, no system of constant number of collective additive tree $r$-spanners can exist for chordal graphs and $r \leq 3$, and no system of constant number of collective additive tree $r$-spanners can exist for weakly chordal graphs and any constant $r$. On the other hand, [10] proved that any unweighted interval graph of diameter $D$ admits an easily constructable system of $2 \log (D-1)+4$ collective additive tree 1-spanners, and any unweighted House-Hole-Domino-free graph with $n$ vertices admits an easily constructable system of at most $2 \log _{2} n$ collective additive tree 2-spanners. Only paper [26] has investigated (so far) collective (multiplicative) tree spanners in the weighted graphs (they were called tree covers there). It was shown that any weighted $n$-vertex planar graph admits a system of $O(\sqrt{n})$ collective multiplicative tree 1-spanners (equivalently, additive tree 0 -spanners) and a system of at most $2 \log _{3 / 2} n$ collective multiplicative tree 3 -spanners.

One of the motivations to introduce this new concept stems from the problem of designing compact and efficient routing schemes in graphs. In [21, 35], a shortest path routing labeling scheme for trees of arbitrary degree and diameter is described that, in total $O(n \log n)$ time, assigns each vertex of an $n$-vertex tree a $O\left(\log ^{2} n / \log \log n\right)$ bit label. Given the label of a source vertex and the label of a destination, it is possible to compute in constant time, based solely on these two labels, the neighbor of the source that heads in the direction of the destination. Clearly, if an $n$-vertex graph $G$ admits a system of $\mu$ collective additive tree $r$-spanners, then $G$ admits a routing labeling scheme of deviation (i.e., additive stretch) $r$ with addresses and routing tables of size $O\left(\mu \log ^{2} n / \log \log n\right)$ bits per vertex. Once computed by the sender in $\mu$ time (by choosing for a given destination an appropriate tree from the collection to perform routing), headers of messages never change, and the routing decision is made in constant time per vertex (for details see $[15,16]$ ).

\subsection{Our Results}

In this paper we generalize and refine the method of [16] for constructing a "small" system of collective additive tree $r$-spanners with small values of $r$ to weighted and larger families of "well" decomposable graphs. We define a large class of graphs, called $(\alpha, \gamma, r)$-decomposable, and show that any weighted $(\alpha, \gamma, r)$ decomposable graph $G$ with $n$ vertices admits a system of at most $\gamma \log _{1 / \alpha} n$ collective additive tree $2 r$-spanners. Then, we show that all weighted planar graphs are $(2 / 3, \sqrt{6 n}, 0)$-decomposable, all weighted graphs with genus at most $g$ are $(2 / 3, O(\sqrt{g n}), 0)$-decomposable, all weighted graphs with tree-width at most $k-1$ are $(1 / 2, k, 0)$-decomposable, all weighted graphs with clique-width at most $k$ are $(2 / 3, k, w)$-decomposable, all weighted graphs with size of largest induced cycle at most $c$ are $(1 / 2,1,\lfloor c / 2\rfloor \mathrm{w})$-decomposable, $(1 / 2,5,\lfloor(c+2) / 3\rfloor \mathrm{w})$-decomposable 
and $(1 / 2,4,(\lfloor c / 3\rfloor+1) w)$-decomposable, and all weighted weakly chordal graphs are $(1 / 2,4, w)$-decomposable. Here and in what follows, w denotes the maximum edge weight in $G$, i.e., w $:=\max \{w(e): e \in E(G)\}$.

As a consequence, we obtain that any weighted planar graph admits a system of $O(\sqrt{n})$ collective additive tree 0 -spanners, any weighted graph with genus at most $g$ admits a system of $O(\sqrt{g n})$ collective additive tree 0 -spanners, any weighted graph with tree-width at most $k-1$ admits a system of $k \log _{2} n$ collective additive tree 0 -spanners, any weighted graph with clique-width at most $k$ admits a system of $k \log _{3 / 2} n$ collective additive tree (2w)-spanners, any weighted graph with size of largest induced cycle at most $c$ admits a system of $\log _{2} n\left(5 \log _{2} n\right.$ and $\left.4 \log _{2} n\right)$ collective additive tree $(2\lfloor c / 2\rfloor w)$-spanners (respectively, $(2\lfloor(c+2) / 3\rfloor w)$-spanners and $(2(\lfloor c / 3\rfloor+1) w)$-spanners $)$, and any weighted weakly chordal graph admits a system of $4 \log _{2} n$ collective additive tree (2w)-spanners. Furthermore, based on this collection of trees, we derive compact and efficient routing schemes for those families of graphs.

\subsection{Basic Notions and Notation}

All graphs considered in this paper are connected, finite, undirected, loopless and without multiple edges. Our graphs can have (non-negative) weights on edges, $w(e)$, $e \in E$, unless specified otherwise. In a weighted graph $G=(V, E)$ the distance $d_{G}(u, v)$ between the vertices $u$ and $v$ is the length of a shortest path connecting $u$ and $v$. If each edge of $G$ has weight 1 , then graph $G$ is called unweighted.

The (open) neighborhood of a vertex $u$ in $G$ is $N(u)=\{v \in V: u v \in E\}$ and the closed neighborhood is $N[u]=N(u) \cup\{u\}$. Define the (hop-)layers of $G$ with respect to a vertex $u$ as follows: $L_{i}(u)=\{x \in V: x$ can be connected to $u$ by a path with $i$ edges but not by a path with $i-1$ edges $\}, i=0,1,2, \ldots$ In a path $P=\left(v_{0}, v_{1}, \ldots, v_{l}\right)$ between vertices $v_{0}$ and $v_{l}$ of $G$, vertices $v_{1}, \ldots, v_{l-1}$ are called inner vertices. Let $r$ be a non-negative real number. A set $D \subseteq V$ is called an $r$ dominating set for a set $S \subseteq V$ of a graph $G$ if $d_{G}(v, D) \leq r$ holds for any $v \in S$.

A tree-decomposition [34] of a graph $G$ is a tree $T$ whose nodes, called bags, are subsets of $V(G)$ such that: (1) $\bigcup_{X \in V(T)} X=V(G)$; (2) for all $\{u, v\} \in E(G)$, there exists $X \in V(T)$ such that $u, v \in X$; and (3) for all $X, Y, Z \in V(T)$, if $Y$ is on the path from $X$ to $Z$ in $T$ then $X \cap Z \subseteq Y$. The width of a tree-decomposition is one less than the maximum cardinality of a bag. Among all the tree-decompositions of $G$, let $T$ be the one with minimum width. The width of $T$ is called the tree-width of the graph $G$ and is denoted by $t w(G)$. We say that $G$ has bounded tree-width if $t w(G)$ is bounded by a constant. It is known that the tree-width of an outerplanar graph and of a series-parallel graph is at most 2 (see, e.g., [4, 28]).

A related notion to tree-width is clique-width. Based on the following operations on vertex-labeled graphs, namely (i) creation of a vertex labeled by integer $l$, (ii) disjoint union (i.e., co-join), (iii) join between all vertices with label $i$ and all vertices with label $j$ for $i \neq j$, and (iv) relabeling all vertices of label $i$ by label $j$, the notion of clique-width $c w(G)$ of a graph $G$ is defined in [20] as the minimum number of labels which are necessary to generate $G$ by using these operations. Clique-width is a complexity measure on graphs somewhat similar to tree-width, but more powerful since every set of graphs with bounded tree-width has bounded clique-width 
[11] but not conversely (cliques have clique-width 2 but unbounded tree-width). It is well-known that the clique-width of a cograph is at most 2 and the clique-width of a distance-hereditary graph is at most 3 (see [25]).

The chordality of a graph $G$ is the size of the largest (in the number of edges) induced cycle of $G$. Define $c$-chordal graphs as the graphs with chordality at most $c$. Then, the well-known chordal graphs are exactly the 3-chordal graphs. An induced cycle of $G$ of size at least 5 is called a hole. The complement of a hole is called an anti-hole. A graph $G$ is weakly chordal if it has neither holes nor anti-holes as induced subgraphs. Clearly, weakly chordal graphs and their complements are 4-chordal. A cograph is a graph having no induced paths on 4 vertices $\left(P_{4} \mathrm{~s}\right)$.

The genus of a graph $G$ is the smallest integer $g$ such that $G$ embeds in a surface of genus $g$ without edge crossings. Planar graphs can be embedded on a sphere, hence $g=0$ for them. A planar graph is outerplanar if all its vertices belong to its outerface.

\section{$2(\alpha, \gamma, r)$-Decomposable Graphs and Their Collective Tree Spanners}

Let $\alpha$ be a positive real number smaller than $1, \gamma$ be a positive integer and $r$ be a non-negative real number. We say that an $n$-vertex graph $G=(V, E)$ is $(\alpha, \gamma, r)$ decomposable if there is a separator $S \subseteq V$, such that the following three conditions hold:

Balanced Separator condition: the removal of $S$ from $G$ leaves no connected component with more than $\alpha n$ vertices;

Bounded $r$-Dominating Set condition: there exists a subset $D \subseteq V$ such that $|D| \leq \gamma$ and for any vertex $u \in S, d_{G}(u, D) \leq r$ (we say that $D r$-dominates $S$ );

Hereditary Family condition: each connected component of the graph, obtained from

$G$ by removing vertices of $S$, is also an $(\alpha, \gamma, r)$-decomposable graph.

Note that, by definition, any graph $G=(V, E)$ having an $r$-dominating set (for $V$ ) of size at most $\gamma$ is $(\alpha, \gamma, r)$-decomposable, for any positive $\alpha<1$. In many cases, in what follows, $D$ will be chosen to be $S$, resulting in 0-domination.

Using these three conditions, one can construct for any $(\alpha, \gamma, r)$-decomposable graph $G$ a (rooted) balanced decomposition tree $\mathcal{B T}(G)$ as follows. If $G$ has an $r$-dominating set of size at most $\gamma$, then $\mathcal{B T}(G)$ is a one node tree. Otherwise, find a balanced separator $S$ with bounded $r$-dominating set in $G$, which exists according to the first and second conditions. Let $G_{1}, G_{2}, \ldots, G_{p}$ be the connected components of the graph $G \backslash S$ obtained from $G$ by removing vertices of $S$. For each graph $G_{i}$ $(i=1, \ldots, p)$, which is $(\alpha, \gamma, r)$-decomposable by the Hereditary Family condition, construct a balanced decomposition tree $\mathcal{B T}\left(G_{i}\right)$ recursively, and build $\mathcal{B T}(G)$ by taking $S$ to be the root and connecting the root of each tree $\mathcal{B T}\left(G_{i}\right)$ as a child of $S$. Clearly, the nodes of $\mathcal{B T}(G)$ represent a partition of the vertex set $V$ of $G$ into clusters $S_{1}, S_{2}, \ldots, S_{q}$, each of them having in $G$ an $r$-dominating set of size at most $\gamma$. For a node $X$ of $\mathcal{B} \mathcal{T}(G)$, denote by $G(\downarrow X)$ the (connected) subgraph of $G$ induced by vertices $\cup\{Y: Y$ is a descendent of $X$ in $\mathcal{B T}(G)\}$ (here we assume that $X$ is a descendent of itself). See Fig. 1 for an illustration.

It is easy to see that a balanced decomposition tree $\mathcal{B T}(G)$ of a graph $G$ with $n$ vertices and $m$ edges has depth at most $\log _{1 / \alpha} n$, which is $O\left(\log _{2} n\right)$ if $\alpha$ is a 


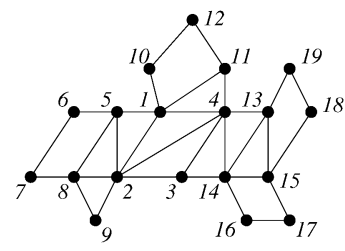

(a)

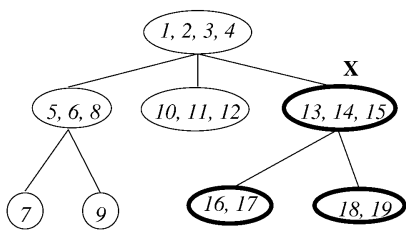

(b)

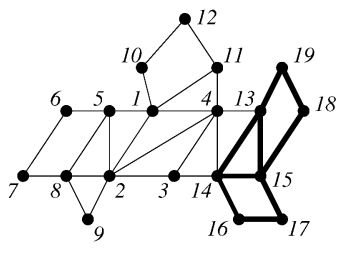

(c)

Fig. 1 (a) A graph $G,($ b) its balanced decomposition tree $\mathcal{B T}(G)$ and (c) an induced subgraph $G(\downarrow X)$ of $G$

constant. Moreover, assuming that a special balanced separator (mentioned above) can be found in polynomial, say $p(n)$, time, the tree $\mathcal{B} \mathcal{T}(G)$ can be constructed in $O\left((p(n)+m) \log _{1 / \alpha} n\right)$ total time.

Consider now two arbitrary vertices $x$ and $y$ of an $(\alpha, \gamma, r)$-decomposable graph $G$ and let $S(x)$ and $S(y)$ be the nodes of $\mathcal{B T}(G)$ containing $x$ and $y$, respectively. Let also $N C A_{\mathcal{B} \mathcal{T}_{(G)}}(S(x), S(y))$ be the nearest common ancestor of nodes $S(x)$ and $S(y)$ in $\mathcal{B T}(G)$ and $\left(X_{0}, X_{1}, \ldots, X_{t}\right)$ be the path of $\mathcal{B T}(G)$ connecting the root $X_{0}$ of $\mathcal{B T}(G)$ with $N C A_{\mathcal{B} \mathcal{T}(G)}(S(x), S(y))=X_{t}$ (in other words, $X_{0}, X_{1}, \ldots, X_{t}$ are the common ancestors of $S(x)$ and $S(y))$. The following lemmata are crucial to our subsequent results.

Lemma 1 Any path $P_{x, y}^{G}$, connecting vertices $x$ and $y$ in $G$, contains a vertex from $X_{0} \cup X_{1} \cup \cdots \cup X_{t}$.

Let $S P_{x, y}^{G}$ be a shortest path of $G$ connecting vertices $x$ and $y$, and let $X_{i}$ be the node of the path $\left(X_{0}, X_{1}, \ldots, X_{t}\right)$ with the smallest index such that $S P_{x, y}^{G} \cap X_{i} \neq \emptyset$ in $G$. Then, the following lemma holds.

Lemma 2 We have $d_{G}(x, y)=d_{G^{\prime}}(x, y)$, where $G^{\prime}:=G\left(\downarrow X_{i}\right)$.

Let $D_{i}$ be an $r$-dominating set of $X_{i}$ in $G^{\prime}=G\left(\downarrow X_{i}\right)$ of size at most $\gamma$. For the graph $G^{\prime}$, consider a set of $\left|D_{i}\right|$ Shortest-Path-trees (SP-trees) $\mathcal{T}\left(D_{i}\right)$, each rooted at a (different) vertex from $D_{i}$. Then, there is a tree $T^{\prime} \in \mathcal{T}\left(D_{i}\right)$ which has the following distance property with respect to those vertices $x$ and $y$.

Lemma 3 For those vertices $x, y \in G\left(\downarrow X_{i}\right)$, there exits a tree $T^{\prime} \in \mathcal{T}\left(D_{i}\right)$ such that $d_{T^{\prime}}(x, y) \leq d_{G}(x, y)+2 r$.

Proof We know, by Lemma 2, that a shortest path $S P_{x, y}^{G}$, intersecting $X_{i}$ and not intersecting any $X_{l}(l<i)$, lies entirely in $G^{\prime}=G\left(\downarrow X_{i}\right)$. Let $x^{\prime}$ be a vertex of $S P_{x, y}^{G} \cap$ $X_{i}$, and denote by $l_{1}$ the distance in $S P_{x, y}^{G}$ between $x$ and $x^{\prime}$ and by $l_{2}$ the distance in $S P_{x, y}^{G}$ between $x^{\prime}$ and $y$. Since $S P_{x, y}^{G}$ is a shortest path of $G$, we have

$$
d_{G}(x, y)=d_{G^{\prime}}(x, y)=l_{1}+l_{2} .
$$


Since $D_{i}$ is an $r$-dominating set of $X_{i}$ in $G^{\prime}$, there exists a vertex $c \in D_{i}$ such that $d_{G^{\prime}}\left(c, x^{\prime}\right) \leq r$. Consider any Shortest-Path-tree $T^{\prime}$ of $G^{\prime}$ rooted at $c$. We have $d_{T^{\prime}}(c, x)=d_{G^{\prime}}(c, x) \leq d_{G^{\prime}}\left(c, x^{\prime}\right)+d_{G}\left(x^{\prime}, x\right) \leq r+l_{1}$. Similarly, $d_{T^{\prime}}(c, y) \leq r+l_{2}$. By triangle inequality, we have

$$
d_{T^{\prime}}(x, y) \leq d_{T^{\prime}}(c, x)+d_{T^{\prime}}(c, y) \leq\left(r+l_{1}\right)+\left(r+l_{2}\right) .
$$

Combining (1) and (2), we obtain $d_{T^{\prime}}(x, y) \leq d_{G}(x, y)+2 r$.

Let now $B_{1}^{i}, \ldots, B_{p_{i}}^{i}$ be the nodes on depth $i$ of the tree $\mathcal{B T}(G)$ and let $D_{1}^{i}, \ldots, D_{p_{i}}^{i}$ be the corresponding $r$-dominating sets. For each subgraph $G_{j}^{i}:=$ $G\left(\downarrow B_{j}^{i}\right)$ of $G\left(i=0,1, \ldots, \operatorname{depth}(\mathcal{B T}(G)), j=1,2, \ldots, p_{i}\right)$, denote by $\mathcal{T}_{j}^{i}$ the set of $S P$-trees of graph $G_{j}^{i}$ rooted at the vertices of $D_{j}^{i}$. Thus, for each $G_{j}^{i}$, we construct at most $\gamma$ Shortest-Path-trees. We call them local subtrees of $G$. Lemma 3 implies

Theorem 1 Let $G$ be an $(\alpha, \gamma, r)$-decomposable graph, $\mathcal{B T}(G)$ be its balanced decomposition tree and $\mathcal{L} \mathcal{T}(G)=\left\{T \in \mathcal{T}_{j}^{i}: i=0,1, \ldots, \operatorname{depth}(\mathcal{B T}(G)), j=\right.$ $\left.1,2, \ldots, p_{i}\right\}$ be its set of local subtrees. Then, for any two vertices $x$ and $y$ of $G$, there exists a local subtree $T^{\prime} \in \mathcal{T}_{j^{\prime}}^{i^{\prime}} \subseteq \mathcal{L} \mathcal{T}(G)$ such that

$$
d_{T^{\prime}}(x, y) \leq d_{G}(x, y)+2 r .
$$

This theorem leads to two import results for the class of $(\alpha, \gamma, r)$-decomposable graphs. Let $G$ be an $(\alpha, \gamma, r)$-decomposable graph with $n$ vertices and $m$ edges, $\mathcal{B T}(G)$ be its balanced decomposition tree and $\mathcal{L} \mathcal{T}(G)$ be the family of its local subtrees (defined above). Consider a graph $H$ obtained by taking the union of all local subtrees of $G$ (by putting all of them together), i.e.,

$$
H:=\bigcup\left\{T: T \in \mathcal{T}_{j}^{i} \subseteq \mathcal{L} \mathcal{T}(G)\right\}=\left(V, \bigcup\left\{E(T): T \in \mathcal{T}_{j}^{i} \subseteq \mathcal{L} \mathcal{T}(G)\right\}\right)
$$

Clearly, $H$ is a spanning subgraph of $G$ and for any two vertices $x$ and $y$ of $G, d_{H}(x, y) \leq d_{G}(x, y)+2 r$ holds. Also, since for any level $i \quad(i=0,1, \ldots$, depth $(\mathcal{B T}(G))$ ) of balanced decomposition tree $\mathcal{B T}(G)$, the corresponding graphs $G_{1}^{i}, \ldots, G_{p_{i}}^{i}$ are pairwise vertex-disjoint and $\left|\mathcal{T}_{j}^{i}\right| \leq \gamma\left(j=1,2, \ldots, p_{i}\right)$, the union $\bigcup\left\{T: T \in \mathcal{T}_{j}^{i}, j=1,2, \ldots, p_{i}\right\}$ has at most $\gamma(n-1)$ edges. Therefore, $H$ has at most $\gamma(n-1) \log _{1 / \alpha} n$ edges in total. Thus, we have proven the following result.

Theorem 2 Any $(\alpha, \gamma, r)$-decomposable graph $G$ with $n$ vertices admits an additive $2 r$-spanner with at most $\gamma(n-1) \log _{1 / \alpha} n$ edges.

Let $\mathcal{T}_{j}^{i}:=\left\{T_{j}^{i}(1), T_{j}^{i}(2), \ldots, T_{j}^{i}(\gamma-1), T_{j}^{i}(\gamma)\right\}$ be the set of $S P$-trees of graph $G_{j}^{i}$ rooted at the vertices of $D_{j}^{i}$. Here, if $p:=\left|D_{j}^{i}\right|<\gamma$ then we can set $T_{j}^{i}(k):=T_{j}^{i}(p)$ for any $k, p+1 \leq k \leq \gamma$. By arbitrarily extending each forest $\left\{T_{1}^{i}(q), T_{2}^{i}(q), \ldots\right.$, $\left.T_{p_{i}}^{i}(q)\right\}(q \in\{1, \ldots, \gamma\})$ to a spanning tree $T^{i}(q)$ of the graph $G$ we can construct at most $\gamma$ spanning trees of $G$ for each level $i(i=0,1, \ldots, \operatorname{depth}(\mathcal{B T}(G)))$ of the 
decomposition tree $\mathcal{B T}(G)$. Totally, this will result into at most $\gamma \times \operatorname{depth}(\mathcal{B T}(G))$ spanning trees $\mathcal{T}(G):=\left\{T^{i}(q): i=0,1, \ldots, \operatorname{depth}(\mathcal{B T}(G)), q=1, \ldots, \gamma\right\}$ of the original graph $G$. Thus, from Theorem 1, we have the following.

Theorem 3 Any $(\alpha, \gamma, r)$-decomposable graph $G$ with $n$ vertices and $m$ edges admits a system $\mathcal{T}(G)$ of at most $\gamma \log _{1 / \alpha} n$ collective additive tree $2 r$-spanners. Moreover, such a system $\mathcal{T}(G)$ can be constructed in $O\left((p(n)+\gamma(m+n \log n)) \log _{1 / \alpha} n\right)$ time, where $p(n)$ is the time needed to find a balanced separator $S$ and its $r$-dominating set $D(|D| \leq \gamma)$ in an $(\alpha, \gamma, r)$-decomposable graph.

From Theorem 3, results of [21, 35] and [16] we conclude.

Corollary 1 Every $(\alpha, \gamma, r)$-decomposable graph $G$ with $n$ vertices admits a routing labeling scheme of deviation $2 r$ with addresses and routing tables of size $O\left(\gamma \log _{1 / \alpha} n \log ^{2} n / \log \log n\right)$ bits per vertex. Once computed by the sender in $\gamma \log _{1 / \alpha} n$ time, headers never change, and the routing decision is made in constant time per vertex.

\section{Particular Classes of $(\alpha, \gamma, r)$-Decomposable Graphs}

\subsection{Graphs Having Balanced Separators of Bounded Size}

In this section we consider graphs that have balanced separators of bounded size. To see that planar graphs are $(2 / 3, \sqrt{6 n}, 0)$-decomposable, we recall the following theorem from [29].

Theorem 4 [29] Let $G$ be any n-vertex planar graph. Then the vertices of $G$ can be partitioned into three sets $A, B, C$, such that no edge joins a vertex in $A$ with a vertex in $B$, neither $A$ nor $B$ has more than $2 / 3 n$ vertices, and $C$ contains no more than $2 \sqrt{2 n}$ vertices. Furthermore $A, B, C$ can be found in $O(n)$ time.

Later, Djidjev [12] improved the constant $2 \sqrt{2}$ to $\sqrt{6}$. Obviously, every connected component of $G \backslash C$ is still a planar graph. This theorem was extended in [2, 13, 22] to bounded genus graphs: a graph $G$ with genus at most $g$ admits a separator $C$ of size $O(\sqrt{g n})$ such that any connected component of $G \backslash C$ contains at most $2 n / 3$ vertices. Moreover, such a balanced separator $C$ can be found in $O(n+g)$ time [2]. Evidently, each connected component of $G \backslash C$ has genus bounded by $g$, too. Hence, the following results follow.

Theorem 5 Every $n$-vertex planar graph is $(2 / 3, \sqrt{6 n}, 0)$-decomposable. Every $n$ vertex graph with genus at most $g$ is $(2 / 3, O(\sqrt{g n}), 0)$-decomposable.

There is another extension of Theorem 4, namely, to the graphs with an excluded minor [3]. A graph $H$ is a minor of a graph $G$ if $H$ can be obtained from a subgraph of $G$ by contracting edges. By an $H$-minor one means a minor of $G$ isomorphic to $H$. Thus the Pontryagin-Kuratowski-Wagner Theorem asserts that planar graphs are those without $K_{5}$ and $K_{3,3}$ minors. The following result was proven in [3]. 
Theorem 6 [3] Let $G$ be an $n$-vertex graph and $H$ be an $h$-vertex graph. If $G$ has no $H$-minor, then the vertices of $G$ can be partitioned into three sets $A, B, C$, such that no edge joins a vertex in $A$ with a vertex in $B$, neither $A$ nor $B$ has more than $2 / 3 n$ vertices, and $C$ contains no more than $\sqrt{h^{3} n}$ vertices. Furthermore $A, B, C$ can be found in $O(\sqrt{h n}(n+m))$ time, where $m$ is the number of edges in $G$.

Since induced subgraphs of an $H$-minor free graph are $H$-minor free, we conclude.

Theorem 7 Let $G$ be an $n$-vertex graph and $H$ be an h-vertex graph. If $G$ has no $H$-minor, then $G$ is $\left(2 / 3, \sqrt{h^{3} n}, 0\right)$-decomposable.

Now we turn to graphs with bounded tree-width. The following theorem is true.

Theorem 8 Every graph with tree-width at most $k$ is $(1 / 2, k+1,0)$-decomposable.

Proof It is well known that if $t w(G)=k$ for a graph $G=(V, E)$, then $G$ can be transformed, by adding new edges, to a chordal graph $G^{+}=\left(V, E^{+}\right)$such that the maximum clique of $G^{+}$is of size $k+1$ (see, e.g., [4, 28]). Moreover, if $k$ is a constant, then the chordal graph $G^{+}$can be constructed in at most $O\left(|V|+\left|E^{+}\right|\right)$time $[4,5]$. In [23] it was shown that every $n$-vertex chordal graph $\Gamma$ contains a maximal clique $C$ such that if the vertices in $C$ are deleted from $\Gamma$, every connected component in the graph induced by any remaining vertices is of size at most $n / 2$. Moreover, according to [23], for any chordal graph on $n$ vertices and $m$ edges, such a separating clique $C$ can be found in $O(n+m)$ time. Applying this result to an $n$-vertex chordal graph $G^{+}$, we obtain a set $S \subseteq V$ of at most $k+1$ vertices such that each connected component of $G^{+} \backslash S$ will have at most $n / 2$ vertices. Since $G$ is a spanning subgraph of $G^{+}$, any connected component of $G \backslash S$ will have at most $n / 2$ vertices, too.

Thus, any graph $G$ with $t w(G)=k$ has a balanced separator consisting of at most $k+1$ vertices. Since induced subgraphs of a graph with tree-width at most $k$ have also tree-width at most $k$ (see, e.g., $[4,28]$ ), the result follows.

Table 1 summarizes the results on collective additive tree spanners of graphs having balanced separators of bounded size. The results are obtained by combining Theorem 3 with Theorems 5, 7 and 8. Note that, for planar graphs, the number of trees in the collection is at most $O(\sqrt{n})$ (not $\sqrt{6 n} \log _{3 / 2} n$ as would follow from Theorem 3 ). This number can be obtained by solving the recurrent formula $\mu(n)=\sqrt{6 n}+\mu(2 / 3 n)$. Similar argument works for other two families of graphs.

Those systems of collective tree spanners described in Table 1 can be constructed in $O((n+\sqrt{n}(m+n \log n)) \log n)=O\left(n^{3 / 2} \log ^{2} n\right)$ time for planar graphs, in $O((n+$ $g+\sqrt{g n}(m+n \log n)) \log n)=O\left(n^{3 / 2} g^{1 / 2} \log ^{2} n\right)$ time for graphs with genus $g$, in $O\left(\left(\sqrt{h n} m+\sqrt{h^{3} n}(m+n \log n)\right) \log n\right)=O\left(h^{3 / 2} n^{1 / 2}\left(m \log n+n \log ^{2} n\right)\right)$ time for graphs without an $h$-vertex minor, and in $O\left(\left(n^{2}+k m+k n \log n\right) \log n\right)$ time for graphs with tree-width at most $k-1$ (for any constant $k \geq 2$ ).

Note that, any shortest path routing labeling scheme in $n$-vertex planar graphs requires at least $\Omega(\sqrt{n})$-bit labels [1]. Hence, by Corollary 1 , there must exist $n$ vertex planar graphs, for which any system of collective additive tree 0 -spanners 
Table 1 Collective additive tree spanners of $n$-vertex $m$-edge graphs having balanced separators of bounded size

\begin{tabular}{llll}
\hline Graph class & $\begin{array}{l}\text { Number of trees } \\
\text { in the collection, } \mu\end{array}$ & $\begin{array}{l}\text { Additive str. } \\
\text { factor, } r\end{array}$ & $\begin{array}{l}\text { Construction } \\
\text { time }\end{array}$ \\
\hline Planar graphs & $O(\sqrt{n})$ & 0 & $O\left(n^{3 / 2} \log ^{2} n\right)$ \\
Graphs with genus $g$ & $O(\sqrt{g n})$ & 0 & $O\left(n^{3 / 2} g^{1 / 2} \log ^{2} n\right)$ \\
Graphs without an $h$-vertex minor & $O\left(\sqrt{h^{3} n}\right)$ & 0 & $O\left(h^{3 / 2} n^{1 / 2}\left(m \log n+n \log ^{2} n\right)\right)$ \\
Graphs with tree-width $k-1$ & $k \log _{2} n$ & 0 & $O\left(\left(n^{2}+k m+k n \log n\right) \log n\right)$ \\
\hline
\end{tabular}

needs to have at least $\Omega\left(\sqrt{n} \log \log n / \log ^{2} n\right)$ trees. We conclude this section with another lower bound, which follows from a result in [10]. Recall that all outerplanar graphs have tree-width at most 2 .

Proposition 1 No system of constant number of collective additive tree $r$-spanners can exist for outerplanar graphs, for any constant $r \geq 0$.

\subsection{Graphs with Bounded Clique-Width}

In this section we will prove that each graph with clique-width at most $k$ is $(2 / 3, k, w)$-decomposable. Recall that $w$ denotes the maximum edge weight in a graph $G$, i.e., $\mathrm{w}:=\max \{w(e): e \in E(G)\}$.

Theorem 9 Every graph with clique-width at most $k$ is $(2 / 3, k, w)$-decomposable.

Proof It was shown in [6] that the vertex set $V$ of any graph $G=(V, E)$ with $n$ vertices and clique-width $c w(G)$ at most $k$ can be partitioned (in polynomial time) into two subsets $A$ and $B:=V \backslash A$ such that both $A$ and $B$ have no more than $2 / 3 n$ vertices and $A$ can be represented as the disjoint union of at most $k$ subsets $A_{1}, \ldots, A_{k}$ (i.e., $\left.A=A_{1} \dot{\cup} \ldots \dot{\cup} A_{k}\right)$, where each $A_{i}(i \in\{1, \ldots, k\})$ has the property that any vertex from $B$ is either adjacent to all $v \in A_{i}$ or to no vertex in $A_{i}$.

Using this, we form a balanced separator $S$ of $G$ as follows. Initially set $S:=\emptyset$, and in each subset $A_{i}$, arbitrarily choose a vertex $v_{i}$. Then, if $N\left(v_{i}\right) \cap B \neq \emptyset$, put $v_{i}$ and $N\left(v_{i}\right) \cap B$ into $S$. Since for any edge $a b \in E$ with $a \in A$ and $b \in B$, vertex $b$ must belong to $S$, we conclude that $S$ is a separator of $G$, separating $A \backslash S$ from $B \backslash S$. Moreover, each connected component of $G \backslash S$ lies entirely either in $A$ or in $B$ and therefore has at most $2 / 3 n$ vertices. By construction of $S$, any vertex $u \in B \cap S$ is adjacent to a vertex from $A^{\prime}:=A \cap S$. As $\left|A^{\prime}\right| \leq k$ and $\mathrm{w}$ is an upper bound on any edge weight, we deduce that $A^{\prime}$ w-dominates $S$ in $G$.

Thus, $S$ is a balanced separator of $G$ and is w-dominated by a set $A^{\prime}$ of cardinality at most $k$. To conclude the proof, it remains to recall that induced subgraphs of a graph with clique-width at most $k$ have clique-width at most $k$, too (see, e.g., [11]), and therefore, by induction, the connected components of $G \backslash S$ induce $(2 / 3, k$, w)decomposable graphs. lary. 
Corollary 2 Any graph with $n$ vertices and clique-width at most $k$ admits a system of at most $k \log _{3 / 2} n$ collective additive tree $2 \mathrm{w}$-spanners, and such a system of spanning trees can be found in polynomial time.

To complement the above result, we give the following lower bound.

Proposition 2 There are (infinitely many) unweighted n-vertex graphs with cliquewidth at most 2 for which any system of collective additive tree 1-spanners will need to have at least $\Omega(n)$ spanning trees.

Proof Consider the complete bipartite graph $G=K_{n, n}$ on $2 n$ vertices. Since $G$ does not have any induced $P_{4}$, it is a cograph. It is known that any cograph has cliquewidth at most 2 (see. e.g., [25]). We show that $G$ will require at least $\Omega(n)$ spanning trees in any system of collective additive tree 1-spanners. Let $\mathcal{T}(G)$ be a system of $\mu$ collective additive tree 1-spanners of $G$. Then, for any two adjacent vertices $x$ and $y$ of $G$ there must exist a spanning tree $T$ such that $d_{T}(x, y) \leq 2$. If $d_{T}(x, y)=2$ then a common neighbor $z$ of $x$ and $y$ in $G$ would form a triangle with vertices $x$ and $y$, which is impossible for $G=K_{n, n}$. Hence, $d_{T}(x, y)=1$ must hold. Thus, every edge $x y$ of $G$ is an edge of some tree $T \in \mathcal{T}(G)$. Since there are $n^{2}$ graph edges to cover by spanning trees from $\mathcal{T}(G)$, we conclude $\mu \geq n^{2} /(2 n-1)>n / 2$.

\subsection{Graphs with Bounded Chordality}

In this section, we consider the class of $c$-chordal graphs and its subclasses. We show that every $c$-chordal graph is $(1 / 2,1,\lfloor c / 2\rfloor w)-,(1 / 2,5,\lfloor(c+2) / 3\rfloor w)$ and $(1 / 2,4,(\lfloor c / 3\rfloor+1) w)$-decomposable, every 4-chordal graph is $(1 / 2,6, w)$ decomposable and every weakly chordal graph is $(1 / 2,4, w)$-decomposable.

In what follows we will need a special ordering of the vertex set of a graph $G=$ $(V, E)$, which refines well known BFS-ordering produced by a breadth-first search. Lexicographic breadth-first search (LexBFS), started at a vertex $u$, orders the vertices of a graph by assigning numbers from $n$ to 1 in the following way. The vertex $u$ gets the number $n$. Then each next available number $k$ is assigned to a vertex $v$ (as yet unnumbered) which has lexically largest vector $\left(s_{n}, s_{n-1}, \ldots, s_{k+1}\right)$, where $s_{i}=1$ if $v$ is adjacent to the vertex numbered $i$, and $s_{i}=0$ otherwise. An ordering of the vertex set of a graph $G$ generated by LexBFS we will call a LexBFS-ordering of $G$, and use $\sigma$ to denote it. The number assigned to a vertex is called LexBFS-ordering number. For any vertex $v, \sigma(v)$ is used to denote its LexBFS-ordering number. For convenience, in the sequel, $\sigma(x)>\sigma(y)$ is simplified as $x>y$. The father of a vertex $v$ is the vertex in $N[v]$ which has the largest LexBFS-ordering number. $f(v)$ is used to denote the father of $v$. LexBFS may be seen to generate a rooted tree $T$ with vertex $u$ as the root.

The following properties of a LexBFS-ordering will be used in what follows (see, e.g., [7, 24]).

(P1) If $x \in L_{i}(u), y \in L_{j}(u)$ and $i<j$, then $x>y$ in $\sigma$.

(P2) If $v \in L_{q}(u)(q>0)$ then $f(v) \in L_{q-1}(u)$ and $f(v)$ is the vertex from $N(v) \cap$ $L_{q-1}(u)$ with the largest number in $\sigma$. 
(P3) If $x>y$, then either $f(x)>f(y)$ or $f(x)=f(y)$.

(P4) If $a<b<c$ and $a c \in E$ and $b c \notin E$ then there exists a vertex $d$ such that $c<d, d b \in E$ and $d a \notin E$.

Note that, properties (P1)-(P3) are guaranteed even by any BFS-ordering. Property (P4), which is the characteristic property of any LexBFS-ordering (see [7]), implies all other three properties and generally is not fulfilled by an arbitrary BFS-ordering. In most cases we will need only properties (P1)-(P3) and hence it would be sufficient to use simply a BFS-ordering of a graph. The full power of LexBFS-orderings (property (P4)) will be used only in the proof of Lemma 7. However, since a LexBFS-ordering of a graph can be easily found in linear time, too (see [24, 27]), we will assume in the sequel that a LexBFS ordering of a graph is given.

Arbitrary c-Chordal Graphs Here, we consider the class of $c$-chordal graphs, $c \geq 3$. We start with an easy consequence of a result from [16].

Theorem 10 Every n-vertex c-chordal graph is (1/2, 1, $\lfloor c / 2\rfloor \mathrm{w})$-decomposable.

Proof In [16], we showed that any $c$-chordal graph has a subset $S \subseteq V$ of vertices computable in $O\left(n^{3}\right)$ time such that any connected component of $G \backslash S$ has at most $n / 2$ vertices and any two vertices $x$ and $y$ of $S$ can be connected in $G$ by a path with at most $\lfloor c / 2\rfloor$ edges. Since in our weighted case any edge has weight at most w, we conclude that in $G$ any vertex $x$ of $S(\lfloor c / 2\rfloor$ w)-dominates $S$. Hence, as induced subgraphs of $c$-chordal graphs are $c$-chordal, the result follows.

Corollary 3 Every $n$-vertex c-chordal graph admits a system of at most $\log _{2} n$ collective additive tree $(2\lfloor c / 2\rfloor \mathrm{w})$-spanners, and such a system of spanning trees can be found in $O\left(n^{3} \log n\right)$ time.

In what follows we will show that every $c$-chordal graph with $c \geq 4$ is also $(1 / 2,5,\lfloor(c+2) / 3\rfloor w)$ - and $(1 / 2,4,(\lfloor c / 3\rfloor+1) w)$-decomposable. To prove these, we first show that any graph has a special balanced separator $S$. Let $N(C):=$ $\bigcup_{v \in C} N(v) \backslash C$ and $N[C]=N(C) \cup C$, for any set $C \subseteq V$.

Lemma 4 Any graph $G$ has a separator $S$ such that each connected component of $G \backslash S$ contains at most $n / 2$ vertices.

Proof Let $\sigma=\left(v_{1}, v_{2}, \ldots, v_{n}\right)$ be a LexBFS-ordering of $G$ and $B_{i}:=\left\{v_{i}, v_{i+1}\right.$, $\left.\ldots, v_{n}\right\}$. Clearly, for any $i=1,2, \ldots, n-1, B_{i}$ is connected. Let $C^{*}(i)$ be a largest (by number of vertices) connected component of $G \backslash B_{i}$. In what follows, $i$ will be chosen to be the largest index such that $\left|V\left(C^{*}(i)\right)\right| \leq n / 2$. Evidently, $i \geq\lceil n / 2\rceil$ and, by maximality of $i$, a largest connected component $C^{*}(i+1)$ of graph $G \backslash B_{i+1}$ must have more than $n / 2$ vertices. It is easy to see that if $C_{1}, C_{2}, \ldots, C_{k}, C^{*}(i+1)$ are the connected components of $G \backslash B_{i+1}$, then the connected components of $G \backslash B_{i}$ will be $C_{1}, C_{2}, \ldots, C_{k}, C_{k+1}, \ldots, C_{k+p}$, where $C_{k+1}, \ldots, C_{k+p}$ are the connected components of the subgraph of $G$ induced by vertices of $C^{*}(i+1) \backslash\left\{v_{i}\right\}$. 


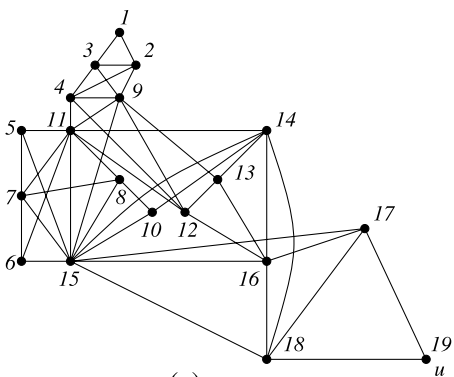

(a)

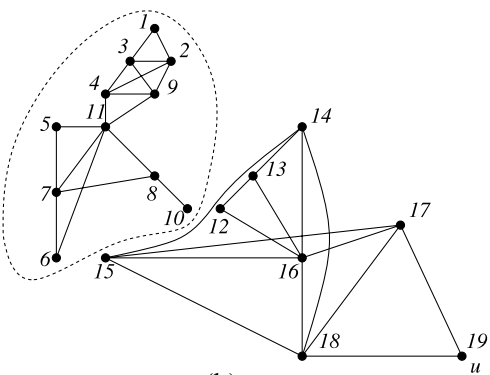

(b)

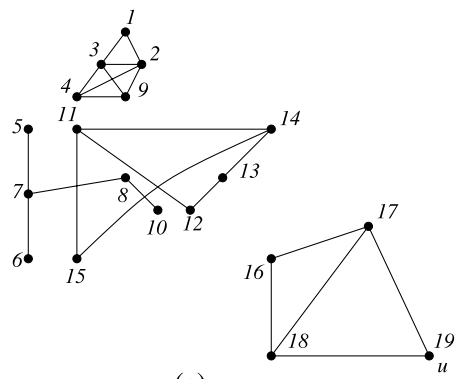

(c)

Fig. 2 (a) A 4-chordal graph $G$ with a LexBFS-ordering. (b) A largest connected component $C^{*}(12)$ of $G \backslash B_{12}$ (circled). A balanced separator $S=\{11,12,13,14,15\}$ and the connected components of $G \backslash S$

Since $\left|V\left(C^{*}(i+1)\right)\right|>n / 2,\left|B_{i+1}\right|+\sum_{i=1}^{k}\left|V\left(C_{i}\right)\right|<n / 2$ holds. Let $C^{\prime}=$ $V\left(C^{*}(i+1)\right), A=B_{i+1} \cap N\left(C^{\prime}\right)$ and $S=A \cup\left\{v_{i}\right\}$. Clearly, all connected components of $G \backslash S$ have at most $n / 2$ vertices as they coincide with components $C_{k+1}, \ldots, C_{k+p}$ and the connected components of the subgraph of $G$ induced by vertices of $C_{1}, C_{2}, \ldots, C_{k}$ and $B_{i+1} \backslash A$.

From the proof of Lemma 4, one can easily design a procedure to find such a balanced separator $S$ in at most $O(|V||E|)$ time. Our goal in this section is to show that in $c$-chordal graphs separator $S$ has a small $r_{c}$-dominating set.

Let $G=(V, E)$ be a $c$-chordal graph with $c \geq 4$ and $\sigma=\left(v_{1}, v_{2}, \ldots, v_{n}\right)$ be a LexBFS-ordering of $G$ (the LexBFS-ordering number of $v_{i}=\sigma(i)$ is $i=\sigma^{-1}\left(v_{i}\right)$ ). For any vertex $x \in V$, define $V_{>x}=\{u \in V: u>x\}$ and $G_{>x}$ to be a subgraph of $G$ induced by $V_{>x}$. Let also $S=A \cup\left\{v_{i}\right\}$ be a separator of $G$ computed as described in the proof of Lemma 4. That is, $C^{*}(i+1)$ is the largest connected components of $G \backslash B_{i+1}$ and $A=N\left(C^{*}(i+1)\right) \cap B_{i+1}$ (see Fig. 2 for an illustration). By the properties of LexBFS-orderings, the following observation clearly holds.

Proposition 3 No vertex of $C^{*}(i+1)$ has a neighbor in $V_{>} f\left(v_{i}\right)$.

We say that a vertex $x$ has the level number $l(x)$ if $x \in L_{l(x)}\left(v_{n}\right)$. Since for any $y \in A, v_{i}<y \leq f\left(v_{i}\right)$ holds, all the vertices of $S$ are either in $L_{l\left(v_{i}\right)}\left(v_{n}\right)$ or in $L_{l\left(v_{i}\right)-1}\left(v_{n}\right)$. Let $S_{1}:=S \cap L_{l\left(v_{i}\right)}\left(v_{n}\right)$ and $S_{2}:=S \cap L_{l\left(v_{i}\right)-1}\left(v_{n}\right)$. 
Lemma 5 There is a set $D$ of at most five vertices in $G$ such that $D(\lfloor(c+2) / 3\rfloor \mathrm{w})$ dominates $S$. Moreover, if $G$ is a c-chordal graph with $4 \leq c \leq 6$, then $D$ consists of only four vertices.

Proof Define vertices $f_{0}, \ldots, f_{a\left(v_{i}\right)}$ as follows: $f_{0}:=v_{i}, f_{1}:=f\left(v_{i}\right), \ldots, f_{a\left(v_{i}\right)}:=$ $f\left(f_{a\left(v_{i}\right)-1}\right)$, where $a\left(v_{i}\right):=\min \left\{\lfloor(c+2) / 3\rfloor, l\left(v_{i}\right)\right\}$. We claim that the set $\left\{f_{1}\right.$, $\left.f_{a\left(v_{i}\right)-1}, f_{a\left(v_{i}\right)}\right\}$ is a $(\lfloor(c+2) / 3\rfloor \mathrm{w})$-dominating set for $S_{1}$.

If $a\left(v_{i}\right)=l\left(v_{i}\right)$ then $f_{a\left(v_{i}\right)}=v_{n}$ and trivially $S_{1}$ is $(\lfloor(c+2) / 3\rfloor \mathrm{w})$-dominated by $f_{a\left(v_{i}\right)}=v_{n}$ since $S_{1} \subseteq L_{a\left(v_{i}\right)}\left(v_{n}\right)$. Therefore, assume that $a\left(v_{i}\right) \neq l\left(v_{i}\right)$, and let $x$ be an arbitrary vertex of $S_{1} \backslash\left\{v_{i}\right\}$. Consider vertices $f_{0}^{\prime}:=x, f_{1}^{\prime}:=f\left(f_{0}^{\prime}\right), \ldots, f_{a\left(v_{i}\right)}^{\prime}:=$ $f\left(f_{a\left(v_{i}\right)-1}^{\prime}\right)$. If there is an index $i\left(0 \leq i<a\left(v_{i}\right)\right)$ such that $f_{i}$ coincides with $f_{i}^{\prime}$ or $f_{i}^{\prime} f_{i} \in E(G)$ or $f_{i}^{\prime} f_{i+1} \in E(G)$, then the distance between $f_{a\left(v_{i}\right)-1}$ (or $f_{a\left(v_{i}\right)}$ ) and $x$ is at most $\lfloor(c+2) / 3\rfloor \mathrm{w}$ and the claim clearly holds. Hence, we may assume that there is no such index $i$. Since $f_{i}<f_{i}^{\prime}$ (by property (P3) of LexBFS-orderings), one concludes that $f_{i} f_{i+1}^{\prime} \notin E(G)$, too, for any $i=0, \ldots, a\left(v_{i}\right)-1$.

Let $P_{G}\left(f_{a\left(v_{i}\right)}, f_{a\left(v_{i}\right)}^{\prime}\right)$ be an induced path between $f_{a\left(v_{i}\right)}$ and $f_{a\left(v_{i}\right)}^{\prime}$ such that, if $\left(f_{a\left(v_{i}\right)}, f_{a\left(v_{i}\right)}^{\prime}\right) \notin E(G)$, then all its inner vertices are from levels $L_{j}, j \leq$ $l\left(f_{a\left(v_{i}\right)}\right)-1$. Let $P_{G}\left(f_{1}, f_{0}^{\prime}\right)$ be an induced path of $G$ obtained by concatenating the two paths $P_{G}\left(f_{1}, f_{a\left(v_{i}\right)}\right):=\left(f_{1}, \ldots, f_{a\left(v_{i}\right)}\right), P_{G}\left(f_{0}^{\prime}, f_{a\left(v_{i}\right)}^{\prime}\right):=\left(f_{0}^{\prime}, \ldots, f_{a\left(v_{i}\right)}^{\prime}\right)$ with $P_{G}\left(f_{a\left(v_{i}\right)}, f_{a\left(v_{i}\right)}^{\prime}\right)$. Obviously, $P_{G}\left(f_{1}, f_{0}^{\prime}\right)$ has at least $\lfloor(c+2) / 3\rfloor-1+\lfloor(c+$ $2) / 3\rfloor+1=2\lfloor(c+2) / 3\rfloor$ edges. Let also $P_{G}^{\prime}\left(f_{1}, f_{0}^{\prime}\right)$ be an induced path between $f_{1}$ and $f_{0}^{\prime}$ all inner vertices of which are from $C^{*}(i+1)$. By construction of $S$, we know that $x>v_{i}$. This and property (P3) of LexBFS-orderings imply that all inner vertices of $P_{G}\left(f_{1}, f_{0}^{\prime}\right)$ are from $V_{>f\left(v_{i}\right)}$. By Proposition 3, no vertex from $V\left(P_{G}^{\prime}\left(f_{1}, f_{0}^{\prime}\right)\right) \backslash\left\{f_{1}, f_{0}^{\prime}\right\}$ is adjacent to a vertex from $V\left(P_{G}\left(f_{1}, f_{0}^{\prime}\right)\right) \backslash\left\{f_{1}, f_{0}^{\prime}\right\}$. Now, by concatenating the two induced paths $P_{G}^{\prime}\left(f_{1}, f_{0}^{\prime}\right)$ and $P_{G}\left(f_{1}, f_{0}^{\prime}\right)$, we obtain a chordless cycle in $G$. Since $G$ is a $c$-chordal graph, the path $P_{G}^{\prime}\left(f_{1}, f_{0}^{\prime}\right)$ cannot have more than $\lfloor(c+2) / 3\rfloor$ edges (otherwise, $G$ will have an induced cycle with at least $c+1$ edges). Hence $d\left(f_{1}, x\right) \leq\lfloor(c+2) / 3\rfloor \mathrm{w}$ and our claim that the set $\left\{f_{1}, f_{a\left(v_{i}\right)-1}, f_{a\left(v_{i}\right)}\right\}$ is a $(\lfloor(c+2) / 3\rfloor \mathrm{w})$-dominating set for $S_{1}$ is proven.

Clearly, if $G$ is a $c$-chordal graph with $4 \leq c \leq 6$, then $a\left(v_{i}\right)$ is at most $2=\lfloor(c+$ $2) / 3\rfloor$. Therefore, in this case, $S_{1}$ is w-dominated by $f_{a\left(v_{i}\right)}=v_{n}$, if $a\left(v_{i}\right)=l\left(v_{i}\right)=1$, or (2w)-dominated by $f_{a\left(v_{i}\right)-1}=f_{1}$ and $f_{a\left(v_{i}\right)}=f_{2}$, if $a\left(v_{i}\right)=\lfloor(c+2) / 3\rfloor=2$.

Let now $v^{\prime}$ be the vertex of $S_{2}$ with the smallest LexBFS-ordering number. Define $a\left(v^{\prime}\right):=\min \left\{\lfloor(c+2) / 3\rfloor, l\left(v_{i}\right)-1\right\}$. Let $f_{0}^{\prime \prime}:=v^{\prime}, f_{1}^{\prime \prime}:=f\left(f_{0}^{\prime \prime}\right), \ldots, f_{a\left(v^{\prime}\right)}^{\prime \prime}:=$ $f\left(f_{a\left(v^{\prime}\right)-1}^{\prime \prime}\right)$. Let $x$ be an arbitrary vertex in $S_{2} \backslash\left\{v^{\prime}\right\}$. Note that, by the definition of $S_{2}$, both $v^{\prime}$ and $x$ have neighbors in $C^{*}(i+1)$. Since $C^{*}(i+1)$ is connected, there is an induced path $P_{G}^{\prime}\left(v^{\prime}, x\right)$ all inner vertices of which are from $C^{*}(i+1)$. Using similar arguments as before, one can show that the set $\left\{v^{\prime}, f_{a\left(v^{\prime}\right)-1}^{\prime \prime}\right\}$ is a $(\lfloor(c+2) / 3\rfloor w)$ dominating set for $S_{2}$.

Set $D:=\left\{v^{\prime}, f_{a\left(v^{\prime}\right)-1}^{\prime \prime}\right\} \cup\left\{v_{i}, f_{a\left(v_{i}\right)-1}, f_{a\left(v_{i}\right)}\right\}$. Clearly, $D$ is a $(\lfloor(c+2) / 3\rfloor \mathrm{w})-$ dominating set for $S$. This concludes the proof of the lemma.

In a similar way we can prove 
Lemma 6 There is a set $D^{\prime}$ of at most four vertices in $G$ such that $D^{\prime}$ is a $((\lfloor c / 3\rfloor+$ 1)w)-dominating set for $S$.

Proof Set $a\left(v_{i}\right):=\min \left\{\lfloor c / 3\rfloor, l\left(v_{i}\right)\right\}$. Let $f_{0}:=v_{i}, f_{1}:=f\left(f_{0}\right), \ldots, f_{a\left(v_{i}\right)}:=$ $f\left(f_{a\left(v_{i}\right)-1}\right)$. We claim that the set $\left\{f_{1}, f_{a\left(v_{i}\right)}\right\}$ is a $((\lfloor c / 3\rfloor+1)$ w)-dominating set of $S_{1}$.

If $a\left(v_{i}\right)=l\left(v_{i}\right)$, then $f_{a\left(v_{i}\right)}=v_{n}$ and claim clearly holds. So, assume $a\left(v_{i}\right) \neq$ $l\left(v_{i}\right)$. Let $x$ be an arbitrary vertex in $S_{1} \backslash\left\{v_{i}\right\}$. Set $f_{0}^{\prime}:=x, f_{1}^{\prime}:=f\left(f_{0}^{\prime}\right), \ldots$, $f_{a\left(v_{i}\right)}^{\prime}:=f\left(f_{a\left(v_{i}\right)-1}^{\prime}\right)$. If there is an index $i, 0 \leq i \leq a\left(v_{i}\right)$, such that $f_{i}=f_{i}^{\prime}$ or $f_{i} f_{i}^{\prime} \in E(G)$ or $f_{i+1} f_{i}^{\prime} \in E(G)$ (for $i<a\left(v_{i}\right)$ ), then we are done. Hence, we may assume that no such $i$ exists. We have also $f_{i} f_{i+1}^{\prime} \notin E(G)$, for any $i=0, \ldots, a\left(v_{i}\right)-1$ since $f_{i}<f_{i}^{\prime}$ holds by property (P3) of LexBFS-orderings. Let $P_{G}\left(f_{a\left(v_{i}\right)}, f_{a\left(v_{i}\right)}^{\prime}\right)$ be an induced path (of length at least 2) between $f_{a\left(v_{i}\right)}$ and $f_{a\left(v_{i}\right)}^{\prime}$ all inner vertices of which are from levels $L_{j}, j \leq l\left(f_{a\left(v_{i}\right)}\right)-1$. By concatenating the paths $P_{G}\left(f_{1}, f_{a\left(v_{i}\right)}\right):=\left(f_{1}, f_{2}, \ldots, f_{a\left(v_{i}\right)}\right), P_{G}\left(f_{0}^{\prime}, f_{a\left(v_{i}\right)}^{\prime}\right):=\left(f_{0}^{\prime}, f_{1}^{\prime}, \ldots, f_{a\left(v_{i}\right)}^{\prime}\right)$ with path $P_{G}\left(f_{a\left(v_{i}\right)}, f_{a\left(v_{i}\right)}^{\prime}\right)$, one gets an induced path with at least $(\lfloor c / 3\rfloor-1)+\lfloor c / 3\rfloor+2=$ $2\lfloor c / 3\rfloor+1$ edges. Since $f_{1} f_{0}^{\prime} \notin E(G)$, there must exist an induced path $P_{G}^{\prime}\left(f_{1}, f_{0}^{\prime}\right)$ between $f_{1}$ and $f_{0}^{\prime}$ all inner vertices of which are from $C^{*}(i+1)$. By Proposition 3 and the fact that $x>v_{i}$, we have also that no inner vertex of $P_{G}^{\prime}\left(f_{1}, f_{0}^{\prime}\right)$ is adjacent to inner vertices of $P_{G}\left(f_{1}, f_{0}^{\prime}\right)$. Therefore, these two paths form an induced cycle. Since $G$ is a $c$-chordal graph, $P_{G}^{\prime}\left(f_{1}, f_{0}^{\prime}\right)$ must have at most $\lfloor c / 3\rfloor+1$ edges. This proves the claim.

Let now $v^{\prime}$ be the vertex with the smallest LexBFS-ordering number in $S_{2}$. Define $a\left(v^{\prime}\right):=\min \left\{\lfloor c / 3\rfloor, l\left(v_{i}\right)-1\right\}$. Let $f_{0}^{\prime \prime}:=v^{\prime}, f_{1}^{\prime \prime}:=f\left(f_{0}^{\prime \prime}\right), \ldots, f_{a\left(v^{\prime}\right)}^{\prime \prime}:=f\left(f_{a\left(v^{\prime}\right)-1}^{\prime \prime}\right)$. Using similar arguments as before, one can show that the set $\left\{f_{0}^{\prime \prime}, f_{a\left(v^{\prime}\right)}^{\prime \prime}\right\}$ is a $((\lfloor c / 3\rfloor+1) w)$-dominating set for $S_{2}$.

Set $D^{\prime}:=\left\{f_{1}, f_{a\left(v_{i}\right)}, f_{0}^{\prime \prime}, f_{a\left(v^{\prime}\right)}^{\prime \prime}\right\}$. Clearly, $D^{\prime}$ is a $((\lfloor c / 3\rfloor+1)$ w)-dominating set for $S$. This completes the proof.

Clearly, for a given $S$, both sets $D$ and $D^{\prime}$ can be found in linear time. Thus, we have proven the following results.

Theorem 11 Let $G$ be a c-chordal graph. Then, $G$ is $(1 / 2,4,\lfloor(c+2) / 3\rfloor \mathrm{w})$ decomposable, if $4 \leq c \leq 6$, and is $(1 / 2,5,\lfloor(c+2) / 3\rfloor w)-$ and $(1 / 2,4,(\lfloor c / 3\rfloor+$ 1)w)-decomposable, if $c>6$.

Corollary 4 Let $G$ be an $n$-vertex and m-edge c-chordal graph. If $c>6$, then $G$ admits a system of at most $5 \log _{2} n$ collective additive tree $(2\lfloor(c+2) / 3\rfloor \mathrm{w})$-spanners and $a$ system of at most $4 \log _{2} n$ collective additive tree $(2(\lfloor c / 3\rfloor+1) w)$-spanners. If $4 \leq c \leq 6$, then $G$ admits a system of at most $4 \log _{2} n$ collective additive tree $(2\lfloor(c+2) / 3\rfloor w)$-spanners. Moreover, such systems of spanning trees can be found in $O(n m \log n)$ time.

From Theorem 10 and Theorem 11 we conclude that any 3-chordal graph is $(1 / 2,1, w)$-decomposable, any 4 -chordal graph or 5 -chordal graph is $(1 / 2,1,2 w)$ decomposable, any 6 -chordal graph is $(1 / 2,1,3 w)$ - and $(1 / 2,4,2 w)$-decomposable, 
any 7 -chordal graph is $(1 / 2,1,3 w)$-decomposable, and any 8 -chordal graph is $(1 / 2,1,4 w)$ - and $(1 / 2,4,3 w)$-decomposable. In the next section we will show that the result for 4 -chordal graphs can be refined. In Table 2 we present our decomposition results for all $c$-chordal graphs.

4-Chordal Graphs Here, we show that every 4-chordal graph is $(1 / 2,6, w)$ decomposable and every weakly chordal graph is $(1 / 2,4, w)$-decomposable.

Let $G=(V, E)$ be a 4-chordal graph and $\sigma=\left(v_{1}, v_{2}, \ldots, v_{n}\right)$ be a LexBFSordering of $G$. Let also $S=A \cup\left\{v_{i}\right\}$ be a separator of $G$ computed as described in the proof of Lemma 4. That is, $C^{*}(i+1)$ is the largest connected components of $G \backslash B_{i+1}$ and $A=N\left(C^{*}(i+1)\right) \cap B_{i+1}$.

Denote by $\bar{C}_{6}$ the complement of an induced cycle $C_{6}$ on 6 vertices. First we will show that any 4-chordal graph not containing $\bar{C}_{6}$ as an induced subgraph is $(1 / 2,4, w)$-decomposable. Clearly, these graphs contain all weakly chordal graphs.

Lemma 7 If $G$ is a 4-chordal graph not containing $\bar{C}_{6}$ as an induced subgraph, then there exists a set $D$ of at most four vertices in $G$ such that $S \subseteq N[D]$.

Proof Let $A^{-}:=\left\{w \in A: w v_{i}, w f\left(v_{i}\right) \notin E(G)\right\}$. We will show that there are at most two vertices $a, b$ in $G$ such that $A^{-} \subseteq N[\{a, b\}]$. Consider a vertex $w \in A^{-}$. Obviously, $w>v_{i}$. By properties (P2) and (P3) of LexBFS-orderings, $f\left(v_{i}\right)<f(w)$ must hold. By Proposition 3, one concludes that $w<f\left(v_{i}\right)$ holds. Let $x \in C^{*}(i+1)$ be a vertex from $N(w)$ which can be connected to $v_{i}$ in $C^{*}(i+1)$ with minimum number of edges.

Claim $1 f\left(v_{i}\right) x \in E(G)$.

Proof The claim can be proved by contradiction. Assume $f\left(v_{i}\right) x \notin E(G)$. Let $P=\left(v_{i}=u_{0}, u_{1}, \ldots, u_{l}=x\right)$ be a path between $v_{i}$ and $x$ in $C^{*}(i+1)$ with minimum number of edges. Clearly, $N(w) \cap P=\{x\}$. Let $u_{l^{\prime}}$ be the vertex of $P$ with largest index which is adjacent to $f\left(v_{i}\right)$. Then path $P_{1}=\left(f\left(v_{i}\right), u_{l^{\prime}}, u_{l^{\prime}+1}, \ldots, u_{l}, w\right)$ is an induced path connecting $f\left(v_{i}\right)$ and $w$, and it consists of at least 3 edges. Since $f\left(v_{i}\right)<f(w)$, there must be an induced path $P_{2}$ between $f\left(v_{i}\right)$ and $w$ all inner vertices of which are from $V_{>f\left(v_{i}\right)}$. Moreover, no vertex from $P_{2}$ can be adjacent to any vertex from $P_{1} \backslash\left\{f\left(v_{i}\right), w\right\}$. Since $P_{2}$ consists of at least 2 edges, by combining $P_{1}$ and $P_{2}$, one gets an induced cycle in $G$ with at least 5 edges. As $G$ is a 4-chordal graph, that is impossible.

Consider a layering $\left\{v_{n}\right\}, L_{1}\left(v_{n}\right), L_{2}\left(v_{n}\right), L_{3}\left(v_{n}\right), \ldots$ of graph $G$, where $L_{i}\left(v_{n}\right)=$ $\left\{x \in V: x\right.$ can be connected to $v_{n}$ by a path with $i$ edges but not by a path with $i-1$ edges\}. Since all the vertices in $B_{i+1}$ have larger LexBFS-ordering numbers than $v_{i}$, by property (P1) of LexBFS-orderings, each vertex in $A^{-}$is either in level $L_{l\left(v_{i}\right)}\left(u_{n}\right)$ or in level $L_{l\left(v_{i}\right)-1}\left(u_{n}\right)$ (recall that $w<f\left(v_{i}\right)$ for any $w \in A^{-}$). Define $A_{u}=\{u: u \in$ $\left.A^{-} \cap L_{l\left(v_{i}\right)}\left(v_{n}\right)\right\}$ and $A_{d}=\left\{u: u \in A^{-} \cap L_{l\left(v_{i}\right)-1}\left(v_{n}\right)\right\}$. Set also $N_{\downarrow}(x):=N(x) \cap$ $\left(L_{l(x)-1}\left(v_{n}\right) \cap V_{>f\left(v_{i}\right)}\right)$ for any $x \in V$. Since for every vertex $w \in A^{-}, f(w)>f\left(v_{i}\right)$ holds, $N_{\downarrow}(w)$ is not empty for any $w \in A^{-}$. We have $l\left(v_{i}\right)>1$, since otherwise, $f\left(v_{i}\right)=v_{n}$ and therefore $w$ must be adjacent to or coincide with $f\left(v_{i}\right)$. 
Claim 2 For any vertex $w \in A^{-}, N_{\downarrow}(w) \subseteq N_{\downarrow}\left(f\left(v_{i}\right)\right)$ holds.

Proof Assume that the statement is not true. Then, one can find a vertex $w^{\prime} \in N_{\downarrow}(w)$ such that $w^{\prime}>f\left(v_{i}\right)$ and $w^{\prime} f\left(v_{i}\right) \notin E(G)$. By Claim 1, there is a vertex $x$ in $C^{*}(i+1)$ which is adjacent to both $f\left(v_{i}\right)$ and $w$. We know also that $x$ is not adjacent to any vertex of $V_{>f\left(v_{i}\right)}$. We distinguish between two cases. First assume $w \in A_{u}$. There must exist an induced path $P_{f\left(v_{i}\right), w^{\prime}}$ between $f\left(v_{i}\right)$ and $w^{\prime}$ such that its inner vertices are all from layers $L_{s}\left(v_{n}\right), s \leq l\left(f\left(v_{i}\right)\right)-1$. This path has at least 2 edges. Moreover, no inner vertex of $P_{f\left(v_{i}\right), w^{\prime}}$ is adjacent to $w$ or $x$. Therefore, paths $\left(f\left(v_{i}\right), x, w, w^{\prime}\right)$ and $P_{f\left(v_{i}\right), w^{\prime}}$ will form a chordless cycle with at least 5 edges in $G$, which is impossible.

Assume now that $w \in A_{d}$. Since $w<f\left(v_{i}\right)<w^{\prime}$ and $w^{\prime} f\left(v_{i}\right) \notin E$ but $w^{\prime} w \in E$, by property (P4) of LexBFS-orderings, there is a vertex $t>w^{\prime}$ such that $t f\left(v_{i}\right) \in$ $E(G)$ and $t w \notin E(G)$. Let $P_{t, w^{\prime}}$ be an induced path connecting $t$ and $w^{\prime}$ all inner vertices of which are from $\bigcup_{i \leq l\left(w^{\prime}\right)-1} L_{i}\left(v_{n}\right) . P_{t, w^{\prime}}$ has at least one edge. Hence, the path $P_{t, w^{\prime}}$ together with $\left(t, f\left(v_{i}\right), x, w, w^{\prime}\right)$ will form an induced cycle with at least 5 edges in $G$, which is impossible.

Claim 3 For any two vertices $w, z \in A_{u}$ or $w, z \in A_{d}$, sets $N_{\downarrow}(w)$ and $N_{\downarrow}(z)$ are comparable.

Proof The claim can be proved by contradiction. Assume $w, z \in A_{u}$ and $N_{\downarrow}(w)$ and $N_{\downarrow}(z)$ are not comparable. Then, there exist two vertices $w^{\prime} \in N_{\downarrow}(w)$ and $z^{\prime} \in N_{\downarrow}(z)$ such that $w^{\prime} z, z^{\prime} w \notin E(G)$. By Claim 2, we know $f\left(v_{i}\right) w^{\prime}, f\left(v_{i}\right) z^{\prime} \in E(G)$. Let $x, y \in C^{*}(i+1)$ be two vertices such that $x w, x f\left(v_{i}\right), y z, y f\left(v_{i}\right) \in E$, the existence of which follows from Claim 1 . As $w^{\prime}, z^{\prime}$ are from $V_{>f\left(v_{i}\right)}$ and $x, y$ are from $C^{*}(i+1)$, there cannon be an edge between sets $\{x, y\}$ and $\left\{z^{\prime}, w^{\prime}\right\}$.

First, we show that both $w z$ and $w^{\prime} z^{\prime}$ must be in $E(G)$. Assume $w^{\prime} z^{\prime} \notin E(G)$. Let $P_{w, z}$ be an induced path between $w$ and $z$ such that all its inner vertices are from $G^{*}(i+1) . P_{w^{\prime}, z^{\prime}}$ is used to denote an induced path between $w^{\prime}$ and $z^{\prime}$ such that its inner vertices are from $\bigcup_{i \leq l\left(w^{\prime}\right)-1} L_{i}\left(v_{n}\right)$. Clearly, the inner vertices of $P_{w^{\prime}, z^{\prime}}$ are not adjacent to any vertex from $P_{w, z}$. Since $P_{w, z}$ has at least one edge and $P_{w^{\prime}, z^{\prime}}$ has at least 2 edges, $P_{w, z}, w w^{\prime}, z z^{\prime}$ and $P_{w^{\prime}, z^{\prime}}$ will form a hole in $G$, which is impossible. This proves that $w^{\prime} z^{\prime}$ must be in $E(G)$. Similarly, if $w z \notin E(G)$, then $P_{w, z}$ has at least 2 edges. Moreover, any inner vertex of $P_{w, z}$ is adjacent neither to $w^{\prime}$ nor to $z^{\prime}$. Hence, $P_{w, z}, w w^{\prime}, w^{\prime} z^{\prime}, z^{\prime} z$ form an induced cycle with at least 5 edges in $G$, which is impossible. Thus, both $w z$ and $w^{\prime} z^{\prime}$ are in $E(G)$.

Second, we claim that neither $w y$ nor $z x$ is in $E(G)$. If $w y \in E(G)$, then since $w z, w^{\prime} z^{\prime} \in E(G)$, vertices $w, y, z, w^{\prime}, z^{\prime}$ and $f\left(v_{i}\right)$ would give an induced $\bar{C}_{6}$ which is also forbidden in $G$. In a similar way, one can show that $z x \in E(G)$ is impossible.

It is easy to see now that vertices $w, z, y, f\left(v_{i}\right), w^{\prime}$ form an induced cycle with 5 edges in $G$. A contradiction obtained proves that $N_{\downarrow}(w)$ and $N_{\downarrow}(z)$ are comparable for any $w, z \in A_{u}$. When $w, z \in A_{d}$, one can show that $N_{\downarrow}(w)$ and $N_{\downarrow}(z)$ are comparable in a similar way. 
Claim 3 ensures that there can be found two vertices $a$ and $b$ in $G$ such that $a \in \bigcap_{w \in A_{u}} N_{\downarrow}(w)$ and $b \in \bigcap_{w \in A_{d}} N_{\downarrow}(w)$. Hence, $A^{-}=A_{d} \cup A_{u}$ is completely contained in $N[\{a, b\}]$, implying $S \subseteq N\left[\left\{v_{i}, f\left(v_{i}\right), a, b\right\}\right]$.

Hence, we have the following results.

Theorem 12 Let $G$ be a 4-chordal graph not containing $\bar{C}_{6}$ as an induced subgraph. Then $G$ is $(1 / 2,4, \mathrm{w})$-decomposable.

Corollary 5 Any n-vertex m-edge 4-chordal graph $G$ not containing $\bar{C}_{6}$ as an induced subgraph (in particular, any weakly chordal graph) admits a system of at most $4 \log _{2} n$ collective additive tree $(2 \mathrm{w})$-spanners. Moreover, such a system of spanning trees can be constructed in $O(n m \log n)$ time.

Note that the class of weakly chordal graphs properly contains such known classes of graphs as interval graphs, chordal graphs, chordal bipartite graphs, permutation graphs, trapezoid graphs, House-Hole-Domino-free graphs, distance-hereditary graphs and many others. Hence, the results of this subsection generalize some known results from $[10,16]$. We recall also that, as it was shown in [10], no system of constant number of collective additive tree $r$-spanners can exist for unweighted weakly chordal graphs for any constant $r \geq 0$.

The above results can easily be extended to all 4-chordal graphs (note that in the proof of Lemma 7 the absence of $\bar{C}_{6}$ in $G$ was important only for Claim 3). We can show that every 4 -chordal graph is $(1 / 2,6, w)$-decomposable.

Lemma 8 If $G$ is a 4-chordal graph, then there exists a set $D$ of at most six vertices in $G$ such that $S \subseteq N[D]$.

Proof Let $A_{u}, A_{d}$ be the same vertex sets as defined in the proof of Lemma 7. Let $x$ be a vertex of $A_{u}$ with minimum $\left|N_{\downarrow}(x)\right|$ among all vertices of $A_{u}$. Similarly, let $y$ be a vertex of $A_{d}$ with minimum $\left|N_{\downarrow}(y)\right|$ among all vertices of $A_{d}$. We claim that for any vertex $z \in A_{u}$, if $x z \notin E(G)$, then $z f(x) \in E(G)$ must hold.

Assume $z f(x) \notin E(G)$ for some $z \in A_{u}$. By the choice of $x$, there must exist a vertex $z^{\prime} \in N_{\downarrow}(z)$ such that $x z^{\prime} \notin E(G)$. Since $x, z$ are in $A_{u}$, there must exist an induced path $P_{G}(x, z)$ all inner vertices of which are from $C^{*}(i+1)$. This path has at least 2 edges. On the other hand, there is a path $P_{G}\left(f(x), z^{\prime}\right)$ in $G$ such that, if $f(x) z^{\prime} \notin E(G)$, then all its inner vertices are from levels $L_{s}\left(v_{n}\right), s<$ $l\left(v_{i}\right)-1$. Path $P_{G}\left(f(x), z^{\prime}\right)$ has at least 1 edge. Furthermore, by Proposition 3 , no vertex on $P_{G}\left(f(x), z^{\prime}\right)$ can be adjacent to inner vertices of $P_{G}(x, z)$. Therefore, $P_{G}\left(f(x), z^{\prime}\right), P_{G}(x, z)$ and two edges $x f(x), z z^{\prime}$ form an induced cycle with at least 5 edges, which is impossible. This contradiction proves our claim.

Analogously, one can show that for any vertex $z \in A_{d}$, if $y z \notin E(G)$, then $z f(y) \in E(G)$ must hold. Now, since $S \subseteq N\left[v_{i}\right] \cup N\left[f\left(v_{i}\right)\right] \cup A_{u} \cup A_{d}$ and $A_{u} \subseteq$ $N[x] \cup N[f(x)], A_{d} \subseteq N[y] \cup N[f(y)]$, we conclude that $S \subseteq N[D]$, where $D:=$ $\left\{v_{i}, f\left(v_{i}\right), x, f(x), y, f(y)\right\}$.

Thus, the following results true. 
Table 2 Summary of the decomposition results obtained for $c$-chordal graphs

\begin{tabular}{ll}
\hline Chordality of the graph & Decomposition results \\
\hline 3 & $(1 / 2,1, \mathrm{w})$ \\
4 & $(1 / 2,1,2 \mathrm{w}),(1 / 2,6, \mathrm{w})$ \\
5 & $(1 / 2,1,2 \mathrm{w})$ \\
6 & $(1 / 2,1,3 \mathrm{w}),(1 / 2,4,2 \mathrm{w})$ \\
7 & $(1 / 2,1,3 \mathrm{w})$ \\
8 & $(1 / 2,1,4 \mathrm{w}),(1 / 2,4,3 \mathrm{w})$ \\
9 & $(1 / 2,1,4 \mathrm{w}),(1 / 2,5,3 \mathrm{w})$ \\
$c \geq 10$ & $(1 / 2,1,\lfloor c / 2\rfloor \mathrm{w}),(1 / 2,4,(\lfloor c / 3\rfloor+1) \mathrm{w})$ \\
$c=3 k, k \geq 4$ & $(1 / 2,1,\lfloor 3 k / 2\rfloor \mathrm{w}),(1 / 2,4,(k+1) \mathrm{w}),(1 / 2,5, k \mathrm{w})$ \\
\hline
\end{tabular}

Theorem 13 Every 4-chordal graph is (1/2, 6, w)-decomposable.

Corollary 6 Any n-vertex m-edge 4-chordal graph $G$ admits a system of at most $6 \log _{2} n$ collective additive tree (2w)-spanners. Moreover, such a system of spanning trees can be constructed in $O(n m \log n)$ time.

Corollary 7 Any n-vertex m-edge 4-chordal graph $G$ admits an additive (2w)spanner with at most $O(n \log n)$ edges. Moreover, such a sparse spanner can be constructed in $O(n m \log n)$ time.

The last result improves and generalizes the known from [9, 16, 31] results on sparse spanners of unweighted chordal graphs.

In Table 2, we summaries all our decomposition results obtained for $c$-chordal graphs.

\section{Conclusion}

In this paper, we continued the approach taken in $[10,15,16,26]$ of studying collective tree spanners of graphs. The method of [16] for constructing a "small" system of collective additive tree $r$-spanners with small values of $r$ was refined and generalized to weighted and larger families of "well" decomposable graphs.

We defined a large class of graphs, called $(\alpha, \gamma, r)$-decomposable, and showed that any weighted $(\alpha, \gamma, r)$-decomposable graph $G$ with $n$ vertices admits a system of at most $\gamma \log _{1 / \alpha} n$ collective additive tree $2 r$-spanners. Using this, we showed that any weighted planar graph admits a system of $O(\sqrt{n})$ collective additive tree 0 -spanners, any weighted graph with genus at most $g$ admits a system of $O(\sqrt{g n})$ collective additive tree 0 -spanners, any weighted graph with tree-width at most $k-1$ admits a system of $k \log _{2} n$ collective additive tree 0 -spanners, any weighted graph with cliquewidth at most $k$ admits a system of $k \log _{3 / 2} n$ collective additive tree (2w)-spanners, any weighted $c$-chordal graph admits a system of $\log _{2} n\left(5 \log _{2} n\right.$ and $\left.4 \log _{2} n\right)$ collective additive tree $(2\lfloor c / 2\rfloor w)$-spanners (respectively, $(2\lfloor(c+2) / 3\rfloor w)$-spanners and 
Table 3 Routing labeling schemes obtained via collective additive tree spanners. The scheme construction time is equal to the time needed to construct an appropriate system of collective additive tree spanners plus $O(n \log n)$ times the number of spanning trees in the system. Thus, the construction time is $O\left(n^{3 / 2} \log ^{2} n\right)$ for planar graphs, $O\left(n^{3 / 2} g^{1 / 2} \log ^{2} n\right)$ for graphs with genus $g, O\left(h^{3 / 2} n^{1 / 2}\left(m \log n+n \log ^{2} n\right)\right)$ for graphs without an $h$-vertex minor, $O\left(\left(n^{2}+k m+k n \log n\right) \log n\right)$ for graphs with tree-width $k-1$, polynomial for graphs with clique-width $k$, and $O(n m \log n)$ for $c$-chordal graphs $(c \geq 4)$

\begin{tabular}{lllll}
\hline $\begin{array}{l}\text { Graph } \\
\text { class }\end{array}$ & $\begin{array}{l}\text { Addresses and } \\
\text { routing tables } \\
\text { (bits per vertex) }\end{array}$ & $\begin{array}{l}\text { Message } \\
\text { initiation } \\
\text { time }\end{array}$ & $\begin{array}{l}\text { Routing } \\
\text { decision } \\
\text { time }\end{array}$ & Deviation \\
\hline Planar & $O\left(\sqrt{n} \log ^{2} n / \log \log n\right)$ & $O(\sqrt{n})$ & $O(1)$ & 0 \\
Of genus $g$ & $O\left(\sqrt{g n} \log ^{2} n / \log \log n\right)$ & $O(\sqrt{g n})$ & $O(1)$ & 0 \\
w/o an $h$-vertex minor & $O\left(\sqrt{h^{3} n} \log ^{2} n / \log \log n\right)$ & $O\left(\sqrt{h^{3} n}\right)$ & $O(1)$ & 0 \\
Of tree-width $k-1$ & $O\left(k \log ^{3} n / \log \log n\right)$ & $k \log _{2} n$ & $O(1)$ & 0 \\
Of clique-width $k$ & $O\left(k \log ^{3} n / \log \log n\right)$ & $k \log _{3} n$ & $O(1)$ & $2 \mathrm{w}$ \\
$c$-chordal $(c \geq 5)$ & $O\left(\log ^{3} n / \log \log n\right)$ & $\log _{2} n$ & $O(1)$ & $2\lfloor c / 2\rfloor \mathrm{w}$ \\
& & $4 \log _{2} n$ & & $2(\lfloor c / 3\rfloor+1) \mathrm{w}$ \\
& & $5 \log _{2} n$ & & $2\lfloor(c+2) / 3\rfloor \mathrm{w}$ \\
4-chordal & $O\left(\log ^{3} n / \log \log n\right)$ & $6 \log _{2} n$ & $O(1)$ & $2 \mathrm{w}$ \\
Weakly chordal & $O\left(\log ^{3} n / \log \log n\right)$ & $4 \log _{2} n$ & $O(1)$ & $2 \mathrm{w}$ \\
\hline
\end{tabular}

$(2(\lfloor c / 3\rfloor+1) w)$-spanners $)$, any weighted 4-chordal graph admits a system of $6 \log _{2} n$ collective additive tree $(2 \mathrm{w})$-spanners, and any weighted weakly chordal graph admits a system of $4 \log _{2} n$ collective additive tree $(2 w)$-spanners.

Combining our decomposition results also with Corollary 1, we obtain the following routing labeling schemes presented in Table 3.

We conclude this paper with few open problems:

(1) Find the complexity of the problem "Given a graph $G$, an integers $\mu$, and a real number $r$, decide whether $G$ has a system of at most $\mu$ collective additive tree $r$ spanners" for different $\mu \geq 1, r \geq 0$ on general graphs and on different restricted families of graphs.

(2) Find better trade-offs between the number of trees $\mu$ and the additive stretch factor $r$ on planar graphs, graphs with genus $g$ and graphs without an $h$-vertex minor.

(3) Find some more applications where collective tree spanners could be useful. The fact that collective tree spanners give a collection of (good) trees might make it easy to adapt many tree algorithms for the graphs that have collective tree $r$ spanners.

\section{References}

1. Abraham, I., Gavoille, C., Malkhi, D.: Compact routing for graphs excluding a fixed minor. In: 19th International Symposium on Distributed Computing (DISC'05). Lecture Notes in Computer Science, vol. 3724, pp. 442-456. Springer, Berlin (2005)

2. Aleksandrov, L., Djidjev, H.: Linear algorithms for partitioning embedded graphs of bounded genus. SIAM J. Discrete Math. 9, 129-150 (1996) 
3. Alon, N., Seymour, P., Thomas, R.: A separator theorem for graphs with an excluded minor and its applications. In: Proceedings of the 22nd Annual ACM Symposium on Theory of Computing, pp. 293-299. Assoc. Comput. Mach., New York (1990)

4. Bodlaender, H.L.: Discovering treewidth. In: SOFSEM 2005: Theory and Practice of Computer Science, 31st Conference on Current Trends in Theory and Practice of Computer Science, January 22-28, 2005. Lecture Notes in Computer Science, vol. 3381, pp. 1-16. Springer, Berlin (2005)

5. Bodlaender, H.L.: A linear-time algorithm for finding tree-decompositions of small treewidth. SIAM J. Comput. 25, 1305-1317 (1996)

6. Borie, R., Johnson, J.L., Raghavan, V., Spinrad, J.P.: Robust polynomial time algorithms on cliquewidth $k$ graphs. Manuscript (2002)

7. Brandstädt, A., Dragan, F.F., Nicolai, F.: LexBFS-orderings and powers of chordal graphs. Discrete Math. 171, 27-42 (1997)

8. Cai, L., Corneil, D.G.: Tree spanners. SIAM J. Discrete Math. 8, 359-387 (1995)

9. Chepoi, V.D., Dragan, F.F., Yan, C.: Additive spanners for $k$-chordal graphs. In: Proceedings of the 5th Conference on Algorithms and Complexity (CIAC 2003), May 28-30, 2003. Lecture Notes in Computer Science, vol. 2653, pp. 96-107. Springer, Berlin (2003)

10. Corneil, D.G., Dragan, F.F., Köhler, E., Yan, C.: Collective tree 1-spanners for interval graphs. In: Proceedings of the 31st International Workshop Graph-Theoretic Concepts in Computer Science (WG'05), June 2005. Lecture Notes in Computer Science, vol. 3787, pp. 151-162. Springer, Berlin (2005)

11. Courcelle, B., Olariu, S.: Upper bounds to the clique-width of graphs. Discrete Appl. Math. 101, 77-114 (2000)

12. Djidjev, H.N.: On the problem of partitioning planar graphs. SIAM J. Algebr. Discrete Methods 3, 229-240 (1982)

13. Djidjev, H.N.: A separator theorem for graphs of fixed genus. Serdica 11, 319-329 (1985)

14. Dragan, F.F., Yan, C.: Collective tree spanners in graphs with bounded genus, chordality, tree-width, or clique-width. In: Proceedings of the 16th Annual International Symposium on Algorithms and Computation (ISAAC 2005), Hainan, China, December 19-21, 2005. Lecture Notes in Computer Science, vol. 3827, pp. 583-592. Springer, Berlin (2005)

15. Dragan, F.F., Yan, C., Corneil, D.G.: Collective tree spanners and routing in AT-free related graphs. J. Graph Algorithms Appl. 10, 97-122 (2006)

16. Dragan, F.F., Yan, C., Lomonosov, I.: Collective tree spanners of graphs. SIAM J. Discrete Math. 20, 241-260 (2006)

17. Elkin, M., Peleg, D.: Approximating $k$-spanner problems for $k>2$. Theor. Comput. Sci. 337, 249-277 (2005)

18. Elkin, M., Peleg, D.: $(1+\epsilon, \beta)$-Spanner constructions for general graphs. SIAM J. Comput. 33, 608631 (2004)

19. Emek, Y., Peleg, D.: Approximating minimum max-stretch spanning trees on unweighted graphs. In: Proceedings of the Fifteenth Annual ACM-SIAM Symposium on Discrete Algorithms (SODA 2004), New Orleans, Louisiana, USA, January 11-14, 2004, pp. 261-270. SIAM, Philadelphia (2004)

20. Engelfriet, J., Rozenberg, G.: Node replacement graph grammars. In: Handbook of Graph Grammars and Computing by Graph Transformation, Foundations, vol. I, pp. 1-94. World Scientific, Singapore (1997)

21. Fraigniaud, P., Gavoille, C.: Routing in trees. In: Proceedings of the 28th Int. Colloquium on Automata, Languages and Programming (ICALP 2001). Lecture Notes in Computer Science, vol. 2076, pp. 757-772. Springer, Berlin (2001)

22. Gilbert, J.R., Hutchinson, J.P., Tarjan, R.E.: A separator theorem for graphs of bounded genus. J. Algorithms 5, 391-407 (1984)

23. Gilbert, J.R., Rose, D.J., Edenbrandt, A.: A separator theorem for chordal graphs. SIAM J. Algebr. Discrete Methods 5, 306-313 (1984)

24. Golumbic, M.C.: Algorithmic Graph Theory and Perfect Graphs. Academic Press, New York (1980)

25. Golumbic, M.C., Rotics, U.: On the clique-width of perfect graph classes. In: Proceedings of the 25th International Workshop Graph-Theoretic Concepts in Computer Science (WG '99), Ascona, Switzerland, June 1999. Lecture Notes in Computer Science, vol. 1665, pp. 135-147. Springer, Berlin (1999)

26. Gupta, A., Kumar, A., Rastogi, R.: Traveling with a pez dispenser (or, routing issues in MPLS). SIAM J. Comput. 34, 453-474 (2005). Appeared also in FOCS 2001 
27. Habib, M., McConnell, R.M., Paul, C., Viennot, L.: Lex-BFS and partition refinement, with applications to transitive orientation, interval graph recognition and consecutive ones testing. Theor. Comput. Sci. 234, 59-84 (2000)

28. Kloks, T.: Treewidth: Computations and Approximations. Lecture Notes in Computer Science, vol. 842. Springer, Berlin (1994)

29. Lipton, R.J., Tarjan, R.E.: A separator theorem for planar graphs. SIAM J. Appl. Math. 36, 346-358 (1979)

30. Liestman, A.L., Shermer, T.: Additive graph spanners. Networks 23, 343-364 (1993)

31. Peleg, D., Schäffer, A.A.: Graph spanners. J. Graph Theory 13, 99-116 (1989)

32. Peleg, D., Ullman, J.D.: An optimal synchronizer for the hypercube. In: Proceedings of the 6th ACM Symposium on Principles of Distributed Computing, Vancouver, pp. 77-85 (1987)

33. Prisner, E., Kratsch, D., Le, H.-O., Müller, H., Wagner, D.: Additive tree spanners. SIAM J. Discrete Math. 17, 332-340 (2003)

34. Robertson, N., Seymour, P.D.: Graph minors II: Algorithmic aspects of tree-width. J. Algorithms 7, 309-322 (1986)

35. Thorup, M., Zwick, U.: Compact routing schemes. In: Proceedings of the 13th Ann. ACM Symp. on Par. Alg. and Arch. (SPAA 2001), pp. 1-10. Assoc. Comput. Mach., New York (2001) 